\title{
Family of Energy Conserving Glossy Reflection Models
}

\author{
Michal Radziszewski and Witold Alda \\ AGH University of Science and Technology, \\ al. Mickiewicza 30, 30-059 Krakow, Poland \\ mradzisz@student.agh.edu.pl, alda@agh.edu.pl
}

\begin{abstract}
We present an improved reflection model optimized for global illumination. The model produces visually plausible images, is symmetric and has improved energy preserving capabilities compared to previous approaches which satisfies these requirements. Having an efficient sampling routine, the model is ready to use in Monte Carlo rendering. Presented model is phenomenological, i.e. it has intuitive glossiness parameter that affects its appearance. Moreover it can be used as a set of basis functions designed to fit material reflection to measured data.
\end{abstract}

Keywords: Reflection Functions, BRDF, Global Illumination, Ray Tracing.

\section{Introduction}

Modeling reflection properties of surfaces is very important for rendering. Traditionally, in global illumination fraction of light which is reflected from a surface is described by a BRDF (Bidirectional Reflection Distribution Function) abstraction. This function is defined over all scene surface points, as well as two light directions - incident and outgoing. As the name suggests, to conform the laws of physics, all BRDFs must be symmetric, i.e. swapping incident and outgoing directions must not change BRDF value. Moreover, the function must be energy preserving - it cannot reflect more light than it receives.

To achieve best results of rendering with global illumination, energy preservation of BRDF should satisfy more strict requirements. It is desirable that basic BRDF model reflects exactly all the light that arrives on a surface. The actual value of reflection is then modeled by a texture. If BRDF is unable to reflect all incident light, even white texture appears to absorb some part of it. In local illumination algorithms this can be corrected a bit by making reflection value more than unit, but in global illumination such trick can have fatal consequences due to multiple light scattering. Our model is strictly energy preserving, while it still maintains other desirable properties.

This paper concentrates on phenomenological glossy BRDFs only. It does not account for wave model of light scattering, neither it touches the concept of BSSRDF. The latter is defined on two directions and two surface points instead of one, which is used to simulate subsurface scattering, during which light is reflected from surface at a point slightly different than is illuminated. 
Paper organization. In the subsequent section there is a brief description of former research related to the BRDF concept. Next, requirements, which should be satisfied by a plausible reflection model are presented. Then there is explained the derivation of our reflection function, followed by comparison of our results with previous ones. Finally, we present a summary, which describes what was achieved during our research and what is left for future development.

\section{Related Work}

The first well known attempt to create glossy reflection is Phong model [1]. This model is, however, neither symmetric nor energy conserving. An improved version of it was created by Neumann et al. 23. Lafortune et al. 4] used combination of generalized Phong reflection functions to adjust scattering model to measured data.

There are popular reflection models based on microfacets. Blinn [5] and Cook et al. [6] assumed that scattering from each individual microfacet is specular, while Oren and Nayar [7] used diffuse reflection instead.

A lot of work was dedicated to anisotropic scattering models. The first well known approach is Kajiya's one 8, which uses physical model of surface reflection. Ward 9] presented a new technique of modeling anisotropic reflection, together with method to measure real-world material reflectances. The Walter's technical report 10 describes how to efficiently implement Ward's model in Monte Carlo renderer. Ashikhmin and Shirley [11] showed how to modify Phong reflection model to support anisotropy.

Some approaches are based on physical laws. He et al. 12 developed a model that supports well many different types of surface reflections. Stam [13] used wave optics to accurately model diffraction of light. Westin et al. 14] used a different approach to obtain this goal. They employed Monte Carlo simulation of scattering of light from surface microgeometry to obtain coefficients to be fitted into their BRDF representation.

On the other hand, Schlick's model 15, is purely phenomenological. It accounts for diffuse and glossy reflection, in isotropic and anisotropic versions through a small set of intuitive parameters. Pellacini et al. 16] used a physically based model of reflection and modified its parameters in a way which makes them perceptually meaningful.

A novel approach of Edwards et al. [17] is designed to preserve all energy while scattering, however at the cost of non-symmetric scattering function. Different approach was taken by Neumann et al. [18. They modified Phong model to increase its reflectivity at grazing angles as much as possible while still satisfying energy conservation and symmetry as well.

Some general knowledge on light reflection models can be found in Lawrence's thesis [19. More information on this topic is in Siggraph Course 20] and in Westin's et al. technical report [21. Westin et al. 22] also provided a detailed comparison of differen BRDF models. Stark et al. 23. shown that many BRDFs can be expressed in more convenient, less than 4D space (two directional vectors). 
Shirley et al. 24] described some general issues which are encountered when reflection models are created.

\section{Properties of Reflection Functions}

In order to create visually plausible images, all reflection functions should satisfy some well defined basic requirements.

Energy conservation. In global illumination it is not enough to ensure that no surface scatters more light that it receives. It is desirable to have a function which scatters exactly all light. We are aware of only Edwards's et al. work [17, which satisfies this requirement, but at the high price of lack of symmetry. Neumann et al. [18] improved reflectivity of Phong model, but the energy preservation still is not ideal.

Symmetry. The symmetry of BRDF is very important when bidirectional methods (which trace rays from viewer and from light as well) are used. When a BRDF is not symmetrical, apropriate corrections similar to described in 25] must be made, in order to get proper rendering results.

Everywhere positive. If a reflection function happens to be equal to zero on part of its domain, the respective surface may potentially render to black, no matter how strong the illumination is. Having a 'blackbody' on the scene is a severe artifact, which is typically mitigated by a complex BRDF with an additional additive diffuse component. However, this option produces dull matte color and is not visually plausible.

Everywhere smooth. Human eye happens to be particularly sensitive on detecting discontinuities of first derivative of illumination, especially on smooth, curved surfaces. This artifact occurs in any BRDF which uses functions such as min or max. Particularly, many microfacet based models use so-called geometric attenuation factor with min function, and look unpleasant at low glossiness values.

Limit \#1 - diffuse. It is very helpful in modeling if glossy BRDF can be made 'just a bit' more glossy than a matte surface. That is, good quality reflection model should be arbitrarily close to matte reflection when glossines is near to zero. Surprisingly, few of BRDF models satisfy this useful and easy to achieve property.

Limit \#2 - specular. Similarly, it is convenient if glossy BRDF becomes near to ideal specular reflection when glossines approaches infinity. Unfortunately, this property is partially much more difficult to achieve than Limit \#1. First, all glossy BRDFs are able to scatter light in near ideal reflection direction, which is correct. Second, energy preservation typically is not satisfied.

Ease of sampling. Having a probability distribution proportional (or almost proportional) to BRDF value, which can be integrated and then inverted analytically, allows efficient BRDF sampling in Monte Carlo rendering. This feature is roughly satisfied in majority of popular BRDF models.

Our work is an attempt to create a BRDF which satisfies all these conditions together. 


\section{Derivation of Reflection Function}

In this section a detailed derivation of the new reflection model is presented. Since this model is purely phenomenological, all mathematical functions chosen to use in it are selected just because of desirable properites they have. This particular choice has no physical basis, and of course, is not unique. Through the rest of this section the notation presented in Table 1 is used. By convention,

Table 1. Notation used in BRDF derivation

\begin{tabular}{cl} 
Symbol & Meaning \\
\hline$f_{r}$ & Reflection function (BRDF) \\
$R$ & Reflectivity of BRDF \\
$\omega_{i}$ & Direction of incident light \\
$\omega_{o}$ & Direction of outgoing light \\
$\omega_{r}$ & Ideal reflection direction of outgoing light \\
$N$ & Surface normal \\
$u, v$ & Arbitrary orthogonal tangent directions \\
$\theta_{i}$ & Angle between $\omega_{i}$ and $N$ \\
$\theta_{r}$ & Angle between $\omega_{r}$ and $N$ \\
$\phi_{i}$ & Angle between $\omega_{i}$ and $u$ \\
$\phi_{r}$ & Angle between $\omega_{r}$ and $u$ \\
$\Omega$ & Hemisphere above surface, BRDF domain
\end{tabular}

all direction vectors are in $\Omega$, i.e. cosine of angle between any of them and $N$ is non-negative. Moreover, these vectors are of unit length.

\subsection{Symmetry and Energy Conservation}

Symmetry requires that incident and outgoing direction can be swapped without modification of BRDF value:

$$
\forall \omega_{i}, \omega_{o} \in \Omega \quad f_{r}\left(\omega_{i}, \omega_{o}\right)=f_{r}\left(\omega_{o}, \omega_{i}\right) .
$$

Energy conservation requires that reflectivity of the BRDF must not be greater than one, and is desirable to be equal to one:

$$
R\left(\omega_{o}\right)=\int_{\Omega} f_{r}\left(\omega_{i}, \omega_{o}\right) \cos \left(\theta_{i}\right) d \omega_{i} \leq 1 .
$$

The reflectivity can be expressed in a different domain. The following expression is used through the rest of this section:

$$
R\left(\theta_{o}, \phi_{o}\right)=\int_{0}^{2 \pi} \int_{0}^{\frac{\pi}{2}} f_{r}\left(\theta_{i}, \phi_{i}, \theta_{o}, \phi_{o}\right) \cos \left(\theta_{i}\right) \sin \left(\theta_{i}\right) d \theta_{i} d \phi_{i} .
$$


It is very useful if reflection function $f_{r}$ can be separated into a product:

$$
f_{r}\left(\theta_{i}, \phi_{i}, \theta_{o}, \phi_{o}\right)=f_{\theta}\left(\theta_{i}, \theta_{o}\right) f_{\phi}\left(\theta_{i}, \phi_{i}, \theta_{o}, \phi_{o}\right),
$$

where $f_{\theta}$ is latitudal reflection and $f_{\phi}$ is longitudal reflection. If $f_{\phi}$ integrates to unit regardless of $\theta_{i}$ and $\theta_{o}$ this separation significantly simplifies reflectivity evaluation, which now can be re-expressed as:

$$
R\left(\theta_{o}, \phi_{o}\right)=\int_{0}^{\frac{\pi}{2}}\left(\int_{0}^{2 \pi} f_{\phi}\left(\theta_{i}, \phi_{i}, \theta_{o}, \phi_{o}\right) d \phi_{i}\right) f_{\theta}\left(\theta_{i}, \theta_{o}\right) \cos \left(\theta_{i}\right) \sin \left(\theta_{i}\right) d \theta_{i},
$$

and energy conservation as:

$$
\int_{0}^{2 \pi} f_{\phi}\left(\theta_{i}, \phi_{i}, \theta_{o}, \phi_{o}\right) d \phi_{i} \leq 1 \quad \text { and } \quad \int_{0}^{\frac{\pi}{2}} f_{\theta}\left(\theta_{i}, \theta_{o}\right) \cos \left(\theta_{i}\right) \sin \left(\theta_{i}\right) d \theta_{i} \leq 1 .
$$

Due to this feature, latitudal and longitudal reflection functions can be treated separately.

\subsection{Latitudal Reflection Function}

The domain of latitudal function is very inconvenient due to the sine and cosine factors in the integrand:

$$
R_{\theta}\left(\theta_{o}\right)=\int_{0}^{\frac{\pi}{2}} f_{\theta}\left(\theta_{i}, \theta_{o}\right) \cos \left(\theta_{i}\right) \sin \left(\theta_{i}\right) d \theta_{i} .
$$

However, substituting $x=\cos ^{2}\left(\theta_{i}\right), y=\cos ^{2}\left(\theta_{o}\right)$ and $d x=-2 \sin \left(\theta_{i}\right) \cos \left(\theta_{i}\right) d \theta_{i}$ leads to much simpler expression for reflectivity:

$$
R_{y}(y)=0.5 \int_{0}^{1} f_{\theta}(x, y) d x .
$$

Despite being much simpler, this space is still not well suited for developing reflection function, mainly because of necessity of symbolic integration. Using the final transformation it may be obtained:

$$
F_{\theta}(x, y)=\int_{0}^{y} \int_{0}^{x} f_{\theta}(s, t) d s d t \quad \text { and } \quad f_{\theta}(x, y)=\frac{\partial^{2} F_{\theta}(x, y)}{\partial x \partial y} .
$$

Designing a function $F_{\theta}$ is much easier than $f_{\theta}$. The requirements that $F_{\theta}$ must satisfy are the following:

$$
\begin{aligned}
\forall_{x, y} \quad F_{\theta}(x, y) & =F_{\theta}(y, x) \\
\forall_{x} \quad F_{\theta}(x, 1) & =x \\
\forall_{x_{1} \leq x_{2}} \quad F_{\theta}\left(x_{1}, y\right) & \leq F_{\theta}\left(x_{2}, y\right)
\end{aligned}
$$


The requirement (11) can be released a bit. If it is not satisfied, it is enough if $F_{\theta}(1,1)=1$ and $F_{\theta}(0,1)=0$ are satisfied instead. In the latter case, applying:

$$
x^{\prime}=F^{-1}(x, 1) \quad \text { and } \quad y^{\prime}=F^{-1}(1, y)
$$

guarantees that $F_{\theta}\left(x^{\prime}, y^{\prime}\right)$ satisfies original requirements (10, 12).

A matte BRDF in this space is expressed as $F_{\theta}=x y$. We have found that the following (unnormalized) function is a plausible initial choice for latitudal glossy reflection:

$$
f_{\theta}(x, y)=\operatorname{sech}^{2}(n(x-y)) .
$$

Transforming this equation into $F_{\theta}$ space leads to:

$$
F_{\theta}(x, y)=\frac{\ln \cosh (n x)+\ln \cosh (n y)-\ln \cosh (n(x-y))}{2 \ln \cosh n} .
$$

This function satisfies only the released requirements, so it is necessary to substitute:

$$
x^{\prime}=\frac{1}{n} \operatorname{artanh}\left(\frac{1-e^{-2 \ln (\cosh n) x}}{\tanh n}\right)
$$

for $x$, and analogical expression for $y$. After substitution and transformation to the $f_{\theta}$ space it may be obtained:

$$
f_{\theta}(x, y)=\frac{m \tanh ^{2} n \cdot e^{-m(x+y)}}{\left(\tanh ^{2} n-\left(1-e^{-m x}\right)\left(1-e^{-m y}\right)\right)^{2}},
$$

where $m=2 \ln \cosh n$. Finally, it should be substituted $x=\cos ^{2}\left(\theta_{i}\right)$ and $y=$ $\cos ^{2}\left(\theta_{r}\right)$. Considering how complex the final expression is, it is clear why it is difficult to guess the form of plausible reflection function, and how useful these auxiliary spaces are.

\subsection{Longitudal Reflection Function}

The longitudal reflection function should be a function of $\cos \left(\phi_{i}-\phi_{r}\right)$. It has to integrate to unit over $[-\pi, \pi]$, so it is reasonable to choose a function that can be integrated analytically:

$$
f_{\phi}\left(\phi_{i}, \phi_{r}\right)=C_{n} \frac{1}{\left[n\left(1-\cos \left(\phi_{i}-\phi_{r}\right)\right)+1\right]^{6}}, \quad \text { and } \quad C_{n}=\frac{(2 n+1)^{5.5}}{2 \pi P_{5}(n)},
$$

where $P_{5}(n)=7.875 n^{5}+21.875 n^{4}+25 n^{3}+15 n^{2}+5 n+1$. When $n=0$, the function becomes constant. When $n$ increases, the function is largest when $\phi_{i}=$ $\phi_{r}$. In the limit, when $n$ approaches infinity, the function converges to $\delta\left(\phi_{i}-\phi_{r}\right)$.

There is still one issue - whenever either $\omega_{i}$ or $\omega_{r}$ is almost parallel to $N, \phi_{i}$ or $\phi_{r}$ is poorly defined. In fact, in these cases, the function should progressively become constant. The simple substitution $n=n^{\prime} \sin \theta_{i} \sin \theta_{r}$ works fine. 


\subsection{Reflection Model}

Combining latitudal and longitudal scattering functions leads to the final BRDF:

$$
\begin{aligned}
f_{r}\left(\theta_{i}, \phi_{i}, \theta_{o}, \phi_{o}\right)= & \frac{\left(2 n_{\phi} \sin \theta_{i} \sin \theta_{r}+1\right)^{5.5}}{2 \pi P_{5}\left(n_{\phi}\right)\left(n_{\phi} \sin \theta_{i} \sin \theta_{r}\left(1-\cos \left(\phi_{i}-\phi_{r}\right)\right)+1\right)^{6}} . \\
& \cdot \frac{m_{\theta} \tanh ^{2} n_{\theta} \cdot e^{-m_{\theta}\left(\cos ^{2} \theta_{i}+\cos ^{2} \theta_{r}\right)}}{\left(\tanh ^{2} n_{\theta}-\left(1-e^{-m_{\theta} \cos ^{2} \theta_{i}}\right)\left(1-e^{-m_{\theta} \cos ^{2} \theta_{r}}\right)\right)^{2}} .
\end{aligned}
$$

The parameters $n_{\theta}$ and $n_{\phi}$ do not have to satisfy $n_{\theta}=n_{\phi}=n$. Using various functions of the form $n_{\theta}=f_{1}(n)$ and $n_{\phi}=f_{2}(n)$ leads to a variety of different glossy scattering models. The reflection angles $\left(\theta_{r}\right.$ and $\left.\phi_{r}\right)$ may be computed from outgoing angles $\theta_{o}$ and $\phi_{o}$ in a few ways, e.g. with ideal reflection, ideal refraction or backward scattering, leading to variety of useful BRDFs.

The reflection model is strictly energy preserving, so cosine weighted BRDF forms probability density functions $(p d f)$ to sample $\theta_{i}$ and $\phi_{i}$ from. Obviously, both $p d f$ s are integrable analytically, which is very helpful.

\section{$5 \quad$ Results}

The following results are generated using a white sphere and a complex dragon model illuminated by a point light source. The proportions of latitudal and longitudal gloss are $n_{\theta}=n$ and $n_{\phi}=0.75 n \sqrt{n \sin \theta_{i} \sin \theta_{r}}$. Fig. 1 examines how selected scattering models cope with little glossiness. Phong-based models expose
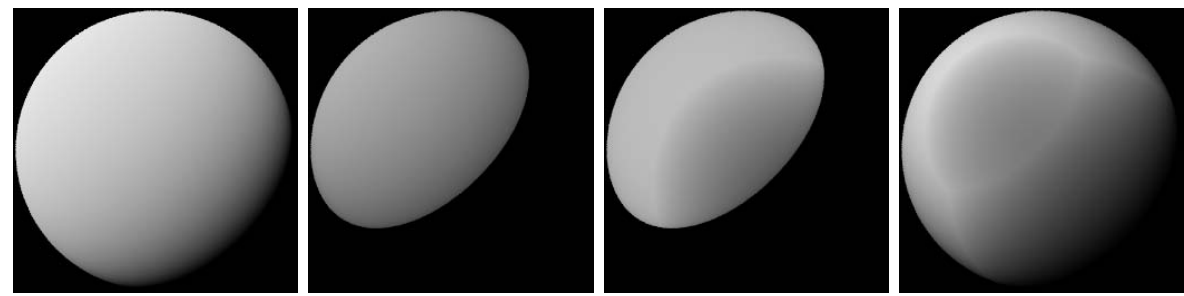

Fig. 1. Comparison of different glossy BRDFs with gloss 'just a bit' more than matte. From left: diffuse reference, reciprocal Phong, max-Phong, microfacet.
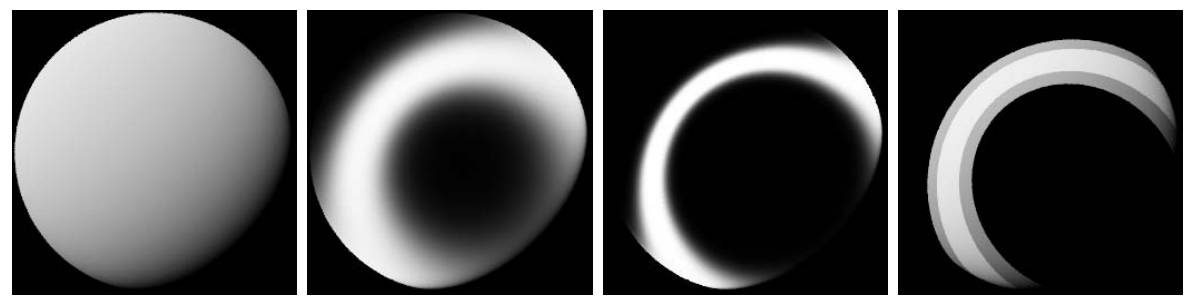

Fig. 2. Latitudal scattering only. From left: glossiness 'just a bit' more than matte, medium glossiness, large glossines, similarity between $\theta_{i}$ and $\theta_{r}$. 

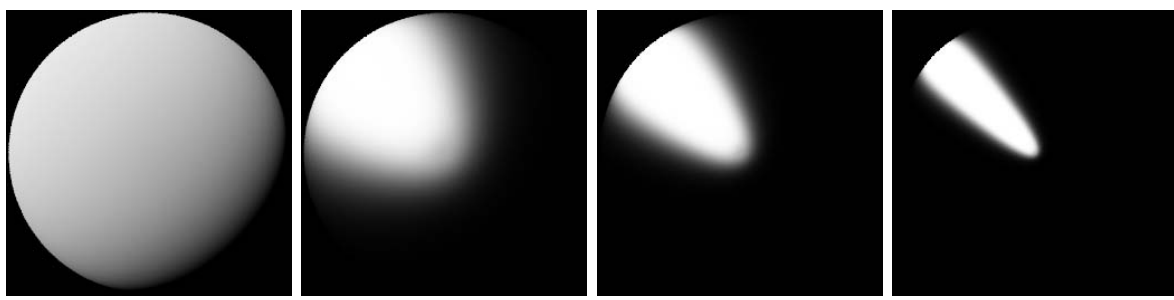

Fig. 3. Longitudal scattering only with varying glossiness
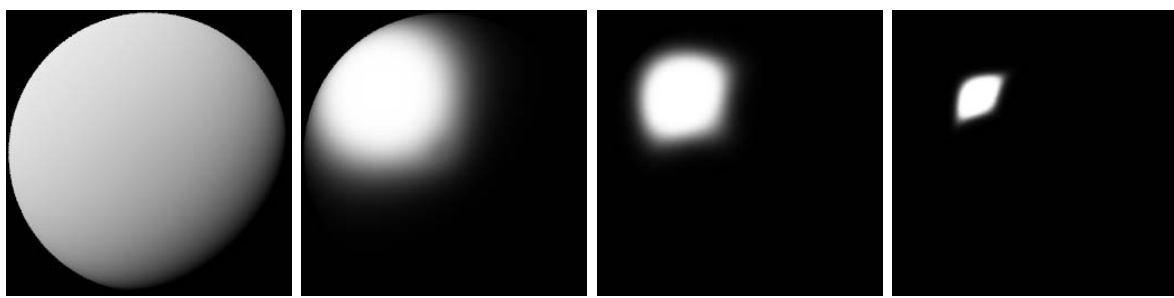

Fig. 4. Product of latitudal and longitudal scattering with increasing glossiness
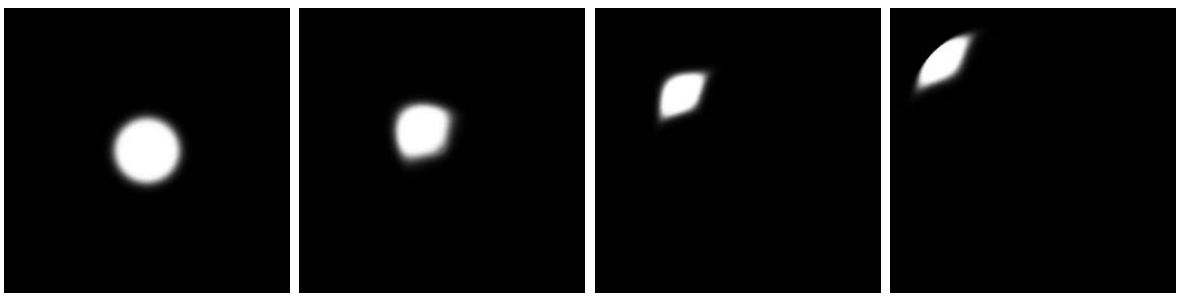

Fig. 5. Scattering with perpendicular (left) and grazing (right) illumination

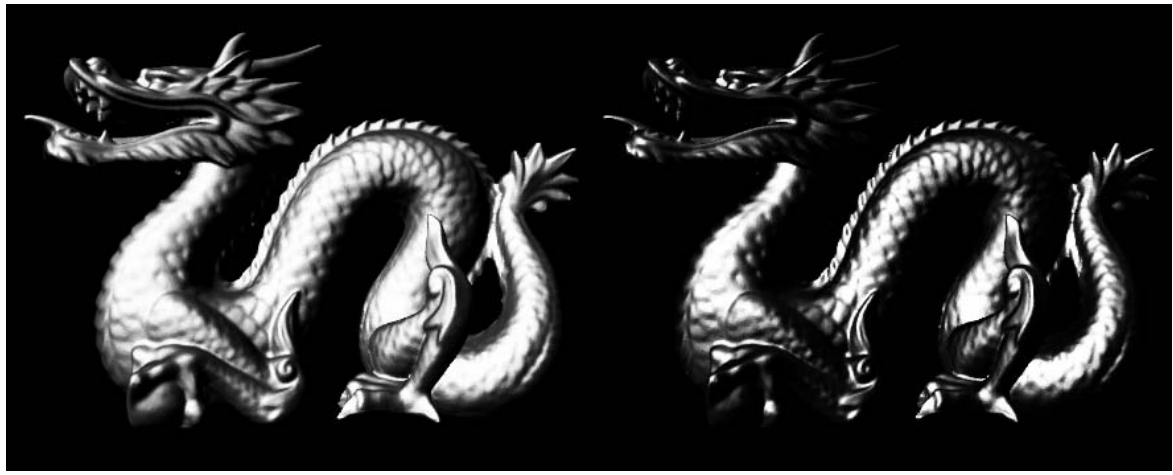

Fig. 6. Complex dragon model rendered with glossiness $n=2$ (left) and $n=4$ (right) 
a zero reflectivity in certain directions, while max-Phong and microfacet models have shading discontinuities. Neither of these models is fully energy conserving.

Fig. 2] shows latitudal component of our reflection model. The scattering is increased at grazing angles to achieve energy conservation. Similarly, Fig. 3 presents longitudal scattering only. In Figs. 4, 5and 6 our BRDF model, defined as a product of latitudal and longitudal scattering is presented. The Fig. 4 shows how the BRDF behaves when glossiness is increased, while the Fig. 5 changes illumination angle using the same glossines. The BRDF exhibits some anisotropy at nonperpendicular illumination, not significant in complex models (Fig. 6).

\section{Conclusions}

We have presented a novel approach to create BRDFs, for which we have designed an energy preserving and symmetrical reflection function. Energy conservation allows improved rendering results. For example, when a model rendered with our function is placed into an environment with uniform illumination, it vanishes. On the other hand, majority of other models lose some energy, especially at grazing angles. For example, Phong reflection tend to absorb much light, producing dark borders around objects, impossible to control. Microfacet model, which conserves energy far better than Phong model, still is not perfect.

In general, properties of our reflection model are different from typically used Phong or microfacet models. It conserves energy and for low glossiness values our model produces neither visual defects like illumination discontinuities, caused by microfacet model, nor black patches, caused by Phong model. Moreover, it smoothly becomes matte, when glossiness approaches zero.

On the other hand, our model produces anisotropy when grazing illumination is encountered, i.e. when viewer direction is rotated around ideal reflection direction, reflection value changes in a way difficult to predict. Phong model does not produce such anisotropy at all, and in microfacet model it is less distracting. Nevertheless, when geometry of illuminated figure is complex, the anisotropy is no more visible, and at grazing angles microfacet model loses numerical stability, especially when $\omega_{i} \approx-\omega_{r}$.

Moreover, simple implementation of latitudal reflection formula causes numerical instabilities when high glossiness is used, i.e. when $n \gtrsim 25$. Currently, our reflection model is best suited for complex geometrical shapes and not extremely high glossiness. In these conditions it produces better results than simple formulae of Phong or microfacet models. Minimizing the impact of anisotropy and numerical instability at high glossiness requires further research in this area. AGH Grant no. 11.11.120.777 is acknowledged.

\section{References}

1. Phong, B.T.: Illumination for computer generated pictures. Communications of the ACM 18(6), 311-317 (1975)

2. Neumann, L., Neumann, A., Szirmay-Kalos, L.: Compact metallic reflectance models. Computer Graphics Forum 18(3), 161-172 (1999) 
3. Neumann, L., Neumann, A., Szirmay-Kalos, L.: Reflectance models with fast importance sampling. Computer Graphics Forum 18(4), 249-265 (1999)

4. Lafortune, E.P., Foo, S.C., Torrance, K.E., Greenberg, D.P.: Non-linear approximation of reflectance functions. In: SIGGRAPH 1997 Proceedings, pp. 117-126 (1997)

5. Blinn, J.F.: Models of light reflection for computer synthesized pictures. In: SIGGRAPH 1977 Proceedings, pp. 192-198. ACM, New York (1977)

6. Cook, R.L., Torrance, K.E.: A reflectance model for computer graphics. ACM Transactions on Graphics 1(1), 7-24 (1982)

7. Oren, M., Nayar, S.K.: Generalization of lambert's reflectance model. In: SIGGRAPH 1994 Proceedings, pp. 239-246. ACM, New York (1994)

8. Kajiya, J.T.: Anisotropic reflection models. In: SIGGRAPH 1985 Proceedings, pp. 15-21. ACM, New York (1985)

9. Ward, G.J.: Measuring and modeling anisotropic reflection. In: SIGGRAPH 1992 Proceedings, pp. 265-272. ACM, New York (1992)

10. Walter, B.: Notes on the ward brdf. Technical Report PCG-05-06, Cornell University (April 2005)

11. Ashikhmin, M., Shirley, P.: An anisotropic phong brdf model. Journal of Graphics Tools 5(2), 25-32 (2000)

12. He, X.D., Torrance, K.E., Sillion, F.X., Greenberg, D.P.: A comprehensive physical model for light reflection. In: SIGGRAPH 1991 Proceedings, pp. 175-186 (1991)

13. Stam, J.: Diffraction shaders. In: SIGGRAPH 1999 Proceedings, pp. 101-110. ACM Press/Addison-Wesley Publishing Co, New York (1999)

14. Westin, S.H., Arvo, J.R., Torrance, K.E.: Predicting reflectance functions from complex surfaces. In: SIGGRAPH 1992 Proceedings, pp. 255-264 (1992)

15. Schlick, C.: A customizable reflectance model for everyday rendering. In: Fourth Eurographics Workshop on Rendering. Number Series EG 93 RW, pp. 73-84 (1993)

16. Pellacini, F., Ferwerda, J.A., Greenberg, D.P.: Toward a psychophysically-based light reflection model for image synthesis. In: SIGGRAPH 2000 Proceedings, pp. 55-64. ACM Press/Addison-Wesley Publishing Co, New York (2000)

17. Edwards, D., Boulos, S., Johnson, J., Shirley, P., Ashikhmin, M., Stark, M., Wyman, C.: The halfway vector disk for brdf modeling. ACM Transactions on Graphics 25(1), 1-18 (2006)

18. Neumann, L., Neumann, A., Szirmay-Kalos, L.: Reflectance models by pumping up the albedo function. Machine Graphics and Vision (1999)

19. Lawrence, J.: Acquisition and Representation of Material Appearance for Editing and Rendering. PhD thesis, Princeton University, Princeton, NJ, USA (2006)

20. Ashikhmin, M., Shirley, P., Marschner, S., Stam, J.: State of the art in modeling and measuring of surface reflection. In: SIGGRAPH 2001 Course \#10 (2001)

21. Westin, S.H., Li, H., Torrance, K.E.: A field guide to brdf models. Technical Report PCG-04-01, Cornell University (January 2004)

22. Westin, S.H., Li, H., Torrance, K.E.: A comparison of four brdf models. Technical Report PCG-04-02, Cornell University (April 2004)

23. Stark, M.M., Arvo, J., Smits, B.: Barycentric parameterizations for isotropic brdfs. IEEE Transactions on Visualization and Computer Graphics 11(2), 126-138 (2005)

24. Shirley, P., Smits, B., Hu, H., Lafortune, E.: A practitioners' assessment of light reflection models. In: Pacific Graphics 1997 Proceedings, pp. 40-50 (1997)

25. Veach, E.: Non-symmetric scattering in light transport algorithms. In: Proceedings of the Eurographics Workshop on Rendering Techniques 1996, pp. 81-90 (1996) 